Beziehung zum Bewohner und wissen, dass sie einen großen Einfluss haben können. Die Atmosphäre wird eine andere, es entwickelt sich unter den Mitarbeitern und Bewohnern eine neue Vertrautheit und mehr Austausch.

\section{Wertschätzung vermitteln}

Sandra Luszai bestätigt das. Die 38-jährige Altenpflegerin arbeitet im evangelischen Altenheim Haus Monheimauf der Demenzstation als Wohnbereichsleiterin. Sie hat sich zur Traumaexpertin ausbilden lassen und leitet eine Wertschätzungsrunde für Demenzerkrankte, die sich einmal wöchentlich trifft. Eine Wertschätzungsgruppe ist ein niedrigschwelliges Angebot. Sie darf nur von Personen geleitet werden, die eine einjährige Ausbildung zur Traumexpertin absolviert haben.

Bei diesem Gruppenangebot können ältere Menschen mit kreativen Mitteln erkunden, was sie in ihrem Leben wertschätzen. Das können sinnliche Erfahrungen über Berührungen sein, Klänge, Musik, Bilder, alte Gegenstände aus der Zeit, in der die Betroffenen jung waren, Steine, Muscheln und Handwerkerzubehör. Die Gegenstände sollen dazu anregen, sich mit den Fragen auseinanderzusetzen: „Was mag ich, welche Farben sprechen mich an, welche Musik höre ich gern, was schätze ich an mir am meisten?" Durch dieses traumasensible Angebot können Menschen mit gerontotraumatologischen Erlebnissen im geschützten Rahmen über ihre Erinnerun- gen sprechen oder sich einfach nur gegenseitig positiv bestärken. Die Erfahrungen in Monheim mit diesen Gruppen sind bislang positiv. Wertschätzung ist deshalb so wichtig, weil viele traumatisierte Menschen darunter leiden, dass ihr Selbstwertgefühl beschädigt wurde.

Die Fortbildung hat Sandra Luszai sensibler und achtsamer im Umgang mit älteren Menschen gemacht. Sie achtet mehr auf Kleinigkeiten, die bei den Bewohnern Angst auslösen könnten: Geht jemand mit Stöckelschuhen über den Flur und erschreckt damit andere? Sind die Vorhänge richtig zugezogen, so dass kein Lichtstrahl nach außen dringt? Warum verhält sich ein Bewohner plötzlich unruhig? War ein Gewitter oder ein Feuerwehralarm der Auslöser?

\section{Sensibel und achtsam}

„Nicht immer“, sagt Sandra Luszai, „haben wir es mit einem Trauma zu tun.“ Man muss sehr genau darauf achten, dass man nicht ein Trauma von früher reaktiviere. „Es geht nicht darum, noch tiefer in der Wunde zu bohren, sondern dem Betroffenen das Gefühl zu geben, dass er verstanden wird, dass es einen emotionalen Halt gibt.“ Das sieht auch Michaela Kulik so. Die Pflegedienstleiterin berichtet von einem Bewohner, der nachts nicht schlafen konnte, weil er sich in seinem Erleben in einer Kriegssituation befand und wenn er eingeschlafen wäre, hätte man ihn erschossen. Diesem Bewohner habe es geholfen, dass sie mit ihm zusammen nachgeschaut habe, ob alles in Ordnung sei. Schließlich habe sie ihm versprochen, dass sie aufpasse und er in Ruhe schlafen könne. „Es ist wichtig, dass alle Mitarbeiter im Haus Bescheid wissen." Sonst könne der Hausmeister nicht verstehen, dass für Bewohner mit kriegstraumatischen Erlebnissen die Vorhänge blickdicht sein müssen (wenn die Tiefflieger einen Lichtstrahl entdeckten, haben sie im Krieg Bomben abgeworfen).

„Wir hatten vor Kurzem eine ältere Dame“, sagt Michaela Kulik, „die zur Kurzzeitpflege bei uns war." Diese Dame hat sich bei uns sehr wohl gefühlt, aber sie wurde sehr ängstlich und orientierungslos, als sie hörte, dass sie für immer bei uns bleiben sollte. Sie erzählte uns, dass sie in der Nachkriegszeit vertrieben wurde. Nun glaubte sie, ein zweites Mal entwurzelt zu werden, weil sie ins Heim eingewiesen werden sollte. Es sei ihnen gelungen, der Dame verständnisvoll zu begegnen. Sie hatten sie ermutigt, sich zuerst in aller Ruhe von Zuhause zu verabschieden, bevor sie ins Heim einziehe. „Wenn wir diese Dame einfach aufgenommen hätten, ohne ihren Hintergrund zu berücksichtigen, hätten wir im Pflegealltag mit ihr Probleme bekommen“, ist Kulik überzeugt. Man hätte dieser alten Dame ein weiteres Mal Unrecht getan, ohne dass das jemandem bewusst geworden wäre. Dieses Verstehen sei auch ein Resultat der Fortbildung im Umgang mit traumatisierten Menschen.

Ingrid Hilgers

\title{
DER MODELLVERSUCH
}

_ Beim Modellversuch „Alter und Trauma - Unerhörtem Raum geben" arbeiten vier Partnereinrichtungen zusammen: Das Diakoniezentrum in Monheim, das Institut für soziale Innovation, der Verein „Wildwasser" und der PariSozial in Minden-Lübbecke.

_ Ab Sommer 2016 stehen die Unterrichtsmaterialien des Vereins Wildwasser kostenfrei im Netz.

_ Die Projektpartner informieren Fach- kräfte und Angehörige, unterstützen Betroffene und sensibilisieren die Öffentlichkeit für das Thema Alter und Trauma. Konzepte zur Traumawürdigung werden in der stationären Altenpflege implementiert und Lernmaterialien für die Ausbildung erstellt.

_ Das Projekt (September 2013 bis August 2016) wird von der Stiftung Wohlfahrtspflege NRW mit 903.600 Euro gefördert. Das Ministerium für
Gesundheit, Emanzipation, Pflege und Alter in NRW (MGEPA) finanziert die wissenschaftliche Begleitung (186.180 Euro).

_ Prof. Dr. Frank Weidner wertet im Deutschen Institut für angewandte Pflegeforschung (dip) Teile des Modellprojekts wissenschaftlich aus. Die Ergebnisse werden im September 2016 vorgestellt.

www.alterundtrauma.de 